\title{
CMB spectral distortions constraints on primordial black holes, cosmic strings and long lived unstable particles revisited
}

\author{
Sandeep Kumar Acharya, ${ }^{a}$ Rishi Khatri ${ }^{a}$ \\ ${ }^{a}$ Department of Theoretical Physics, Tata Institute of Fundamental Research, Mumbai 400005, India \\ E-mail: sandeepkumar@theory.tifr.res.in, khatri@theory.tifr.res.in
}

\begin{abstract}
We calculate the spectral distortions from Hawking evaporation of primordial black holes before the epoch of recombination, taking into account emission of all standard model particles, including quark and gluons, and evolving the resulting particle cascades in the expanding Universe. We show that the constraints on the abundance of primordial black holes are stronger by more than an order of magnitude compared to the previous calculations which take only the primary photon emission into account. We also show that the shapes of the spectral distortions is different from the $y$ or $i$-type distortions and are sensitive to the mass of the primordial black holes. We also extend previous constraints on the decay of long lived unstable particles before recombination to additional decay channels. We show that for dark matter mass $\lesssim 1 \mathrm{GeV}$, the spectral distortion shape is a function of the dark matter mass as well as the decay channel to standard model particles. We also provide new spectral distortion constraints on superconducting cosmic string decay. We explicitly show that consideration of emitted photon spectrum from string decay is not only important for the future experiments but also for already available COBE-FIRAS data.
\end{abstract}




\section{Introduction}

Spectral distortions of the cosmic microwave background (CMB), abundance of light elements produced in the big bang nucleosynthesis (BBN), and CMB anisotropy power spectrum provide multiple avenues to constrain external electromagnetic energy injection into the primordial baryon-photon fluid in the early universe. Injection of energy around the recombination era $(z \sim 1000)$ modifies the standard recombination history, damping the CMB temperature anisotropy power spectrum while boosting the polarization power spectrum [13]. Recently, it was shown that the CMB anisotropies can constrain energy injection at redshifts as high as $z \sim 10000$ [4]. CMB temperature anisotropy power spectrum has been measured to exquisite precision by Planck [5] and ground based experiments such as SPT [6], and ACT [7]. Next generation experiments [8-12], with more precise polarisation measurement, can strengthen the present day constraints by an order of magnitude. Energetic photons in the electromagnetic cascades, triggered by the injection of high energy particles, can photo-dissociate light elements produced by BBN, changing their abundances. BBN can constrain energy injection upto very high redshifts [13-15]. BBN constraints, limited by the astrophysical uncertainties on the measurement of the abundance of light elements, are well established [4, 16-19] and unlikely to improve significantly in the near future. The importance of consistent evolution of electromagnetic cascades when calculating BBN constraints was emphasized recently in [4].

The best measurement of the CMB spectrum was done more than 25 years ago by COBE-FIRAS (Cosmic Background Explorer- Far InfraRed Absolute Spectrophotometer) $[20,21]$. In contrast to the CMB anisotropies and the BBN, the spectral distortion constraints have the potential for many orders of magnitude improvement with near future experiments like PIXIE [9]. At $z \gtrsim 2 \times 10^{6}$, photon non-conserving processes such as double Compton scattering and bremsstrahlung together with Compton scattering, establish a Planck spectrum and exponentially suppress any departure from equilibrium. Any energy injection above $z \gtrsim 2 \times 10^{6}$ in the form of heating, therefore, raises the temperature of the photons while preserving the blackbody spectrum [22-25]. At $z \lesssim 2 \times 10^{6}$, energy injection can give rise to $y, \mu$ or $i$-type distortions $[22,24,26-30]$. Previous calculations assumed that all of the injected energy goes into heating up the background electrons which boost the CMB photons by non-relativistic Compton scattering. Recently, it was shown that for high energy (>10 $\mathrm{keV}$ ) electron-positron and photon injections, significant relativistic corrections are expected [31]. Relativistic electrons and positrons produced during the particle cascade, triggered by the high energy injected particle, can boost the CMB photons to significantly higher energy compared to the $y$-type distortion, imprinting characteristic energy dependence on spectral distortion shapes due to relativistic collision processes. These ultra-relativistic distortions modify the constraints on energy injections. It was shown in [32] that for dark matter decay to monochromatic electron-positron pairs or photon pairs, the constraints can relax by upto a factor of 4-5 for injection redshifts $z_{\text {inj }}<50000$. In this paper, we extend these calculations to additional dark matter decay channels, Hawking evaporation of primordial black holes and 
decay of cosmic strings.

There are many particle physics models beyond the standard model which can provide a dark matter candidate. The simplest candidate is the WIMP (Weakly Interacting Massive Particle) which invokes just one extra particle with weak interaction to explain the present density of dark matter. However, in general, in theories such as supersymmetric and Kaluza-Klein type extra-dimensional models, there are large number of new particles with the lightest stable particle being the dark matter candidate. A detailed discussion of possible dark matter candidates can be found in reviews such as [33, 34]. These models are invoked not only to explain dark matter but also to explain problems related to standard model physics, for example, the hierarchy problem. These models typically include small coupling with standard model particles so as not to violate the collider and astrophysics constraints while simultaneously alleviating problems related to particle physics. In these models, it is perfectly reasonable to assume that many new particles were produced in the early Universe. The lightest stable particle is the dark matter today. However, there could have been additional particles which were unstable and decayed later into standard model particles and/or dark matter particles. If the lifetime of these new particles is larger than $\sim 2$ months (decays happening at $z \lesssim 2 \times 10^{6}$ ), then a fraction of energy going into the standard model particles will give rise to $\mathrm{CMB}$ spectral distortions.

Another interesting scenario, which can inject energetic electromagnetic particles at high redshifts, is Hawking evaporation of primordial black holes and accreting primordial black holes. We will only consider evaporting primordial black holes in this paper, ignoring accretion. Accretion can release additional energy and make the constraints stronger, especially towards the higher mass end, albeit with significant uncertainties related to the modeling [35-37]. Evaporating black holes emit energetic standard model particles having energies of the order of the temperature of the black hole which in turn depends on the mass of the black hole $[38,39]$. The shape of the spectrum of emitted particles at energies much larger compared to the black hole temperature is thermal. However, at low and intermediate energies, there can be significant deviation from the thermal spectrum [40, 41]. Black holes with temperature $\gtrsim 1 \mathrm{GeV}$ emit mostly quarks and gluons which produce significant amount of secondary electrons, positrons and photons after hadronization [42]. Spectral distortion constraints for primordial black holes with mass range $\sim 10^{11} \mathrm{~g}-10^{13} \mathrm{~g}$ (corresponding to black hole evaporation between y and $\mu$ era) have previously been studied in [43]. However, they only considered the primary photon emission from the black holes and ignored emission of other particles. Recent work of [44] takes into account all particles but assumes that all energy goes into non-relativistic $y, i$ and $\mu$-type distortions (referred collectively hereafter as the yim distortions). Black holes emit all particles democratically provided the emission is kinematically allowed i.e. the temperature of the evaporating black hole is of the same order or larger compared to the mass of the emitted particle. The fractions of energy going into different particles are proportional to the multiplicity of each particle. For example, 6 quarks with 3 colors, antiparticles and spins of $1 / 2$ have multiplicity 72 and account for the bulk of emitted energy while the photons with multiplicity of 2 corresponding to 2 polarizations 
account for a sub-dominant fraction of evaporating energy. Therefore, it is important to take into account secondary photon, electron, and positron emission from quarks and gluons at high temperatures where primary photon emission only accounts for a tiny fraction of the total emitted energy. In this work, we take into account all the particles emitted by black holes and calculate the non-thermal (ntr) spectral distortion shape by evolving the electromagnetic cascades. We compare the full non-thermal distortion spectrum with thermal distortion spectrum (yim distortion). For black hole evaporation at $z \lesssim 10^{5}$, the shape of non-thermal distortion is significantly different from that of thermal non-relativistic spectral distortions, while for higher redshifts, spectral distortions are thermalized to $\mu$ distortion.

Phase transitions in the early universe can give rise to topological defects like monopoles, strings and domain walls [45]. String theory also predicts the existence of such objects [46]. These cosmic strings carry energy, source perturbations in surrounding matter, and thus, can have observable effects in the CMB anisotropy power spectrum [47, 48]. Cosmic strings can be superconducting and can decay by emitting their energy as high energy electromagnetic particles [49] and can therefore create CMB spectral distortions. Since the spectrum of photons emitted by the superconducting cosmic strings is highly non-thermal and depends on the cosmic string tension and current, we expect that the shape of the resulting CMB spectral distortions to also be different from the yim distortions and in particular be sensitive to the parameters of the cosmic string.

We use the COBE-FIRAS [20] data to give constraints for different energy injection scenarios using CMB spectral distortions calculated by evolving the electromagnetic cascades. In particular, we do not use the yim approximation but use the actual spectral distortion shapes. We also give forecasts for a future PIXIE-like experiment assuming a factor of 1000 enhancement in the sensitivity over COBE-FIRAS. We use Planck [5] $\Lambda$ CDM cosmological parameters for all calculations.

\section{Electromagnetic cascades in the expanding Universe}

In this section we briefly explain the qualitative aspects of electromagnetic cascade evolution. The technical details can be found in [31]. We follow the computational approach of [50-52] in an ionized universe as described in [31]. We divide the energy range of interest into 500 logarithmically-spaced energy bins for each particle (e.g. photons, electrons and positrons) in dimensionless frequency variable $x=E / T_{\mathrm{CMB}}$, where $E$ is the kinetic energy of the electron or positron or the energy of the photon, and $T=2.725(1+z) \mathrm{K}$ is the CMB temperature. A high energy injected particle, after repeated scattering, deposits its energy by boosting the background particles. In an ionized universe, electrons with energy below keV deposit their energy via heat while higher energy electrons boost the CMB photons by inverse Compton scattering process (ICS). Positrons can release two photons with energy $511 \mathrm{keV}$ after annihilating with the background electrons. The rate of energy deposition for electrons and positrons is much faster compared to the Hubble rate (Fig. 2 of [31]) and it is a very good approximation to assume that the electrons and positrons deposit their energy instantaneously. 
Photons on the other hand deposit their energy at a comparable or slower rate compared to the expansion rate and the photon spectrum needs to be evolved by taking the background expansion into account. We take into account collision processes such as Compton scattering, photon-photon elastic scattering and electron-positron pair production. We are essentially solving a system of coupled differential equations (Eq. 3.1 of [31]) where the particles in the cascade move from one energy bin to another as the cascade evolves. The probabilities or rates of transfer between the energy bins are determined by the competition between the different collision processes and the expansion rate of the universe. These particles in the cascade have relativistic energies with non-thermal distribution. The shape of the CMB spectral distortions from these non-thermal particles is significantly different from the $y$-type and the $i$-type distortions. For non-thermal photons, with energy much higher compared to the CMB photons, we can ignore the energy distribution of the background electrons. For photons with energy comparable to the CMB photons, we take into account Doppler broadening due to the thermal motion of the background electrons. We also take into account stimulated Compton scattering at photon energies comparable to the background CMB photons. At $z \gtrsim 10^{5}$, the non-thermal relativistic CMB spectral distortions also thermalize to $\mu$-type distortion [32] and become a function of only the total injected energy, losing sensitivity to the initial spectrum of injected particles.

\section{Primordial black hole evaporation}

Primordial black holes are formed from high energy density peaks in the early universe when overdense regions decouple from the Hubble expansion and collapse under their self-gravity [53-55]. If the density perturbation at the stage of horizon entry exceeds a threshold, determined by the thermal pressure, it can lead to the formation of a black hole with mass of the order of horizon mass, $M_{\mathrm{BH}} \sim \frac{c^{3} t}{G}$ [56], where t is the cosmological time, G is Newton's gravitational constant and $\mathrm{c}$ is the speed of light. Depending on the formation epoch, primordial black holes can span huge range of mass, from Planck scale to hundreds of solar mass. We will consider only Schwarzschild black holes in this paper. This is a good approximation because for a rotating black hole more than 50 percent of black hole energy is emitted when the black hole has already lost most of its spin and is slowly rotating [57].

Black holes, once formed, radiate particles as a hot body with temperature (with $c=\hbar=$ $\left.k_{B}=1\right)[38,56]$,

$$
T_{\mathrm{BH}}=\frac{1}{8 \pi G M_{\mathrm{BH}}}=1.06 M_{10}^{-1} \mathrm{TeV}
$$

where $M_{10}=M_{\mathrm{BH}} / 10^{10} \mathrm{~g}$ is the mass of black hole in units of $10^{10} \mathrm{~g}$.

The lifetime of evaporating black holes is approximately given by [42, 56],

$$
t_{\mathrm{BH}}=407\left[\frac{f\left(M_{\mathrm{BH}}\right)}{15.35}\right]^{-1} M_{10}^{3} \mathrm{~s},
$$

where $f\left(M_{\mathrm{BH}}\right)$ carries the information about the emitted particles and is defined below. The particle emission rate from the Schwarzschild black holes in the energy interval $\mathrm{E}$ and $\mathrm{E}+\mathrm{dE}$ 
is given by $[40,41]$,

$$
\frac{d N}{d t}=\frac{\Gamma_{s}\left(E, M_{\mathrm{BH}}, m\right)}{2 \pi} \frac{1}{e^{E / T_{\mathrm{BH}}}-(-1)^{2 s}} d E,
$$

where $\Gamma_{s}$ is the absorption coefficient which depends on the spin $(s)$ and mass $(m)$ of the emitted particle and the mass of the black hole [40,41]. The spectrum of emitted particles just outside the black hole horizon has a thermal distribution. These particles experience an effective potential outside the horizon which is determined by the mass of black hole and the angular momentum and mass of the emitted particle [58]. Therefore, the emission spectrum of the particles far way from the horizon becomes mass- and spin-dependent. These absorption coefficients become mass (of particle) and spin-independent at high energy approaching the limit, $\Gamma_{s}\left(E / T_{\mathrm{BH}}>>1\right)=27 G^{2} M^{2}$. The PBH mass loss rate can be written as [42],

$$
\frac{d M_{\mathrm{BH}}}{d t}=-5.34 \times 10^{25} \frac{f\left(M_{\mathrm{BH}}\right)}{M_{\mathrm{BH}}^{2}} \mathrm{gs}^{-1} .
$$

where $f\left(M_{\mathrm{BH}}\right)$ is approximately given by,

$$
\begin{array}{r}
f\left(M_{\mathrm{BH}}\right)=1.569+0.569\left[\exp \left(-\frac{M_{\mathrm{BH}}}{x_{\mathrm{bh}, s=1 / 2} M_{\mu}^{T}}\right)+3 \exp \left(-\frac{M_{\mathrm{BH}}}{x_{\mathrm{bh}, s=1 / 2} M_{u}^{T}}\right)+3 \exp \left(-\frac{M_{\mathrm{BH}}}{x_{\mathrm{bh}, s=1 / 2} M_{d}^{d}}\right)\right. \\
+3 \exp \left(-\frac{M_{\mathrm{BH}}}{x_{\mathrm{bh}, s=1 / 2} M_{s}^{T}}\right)+3 \exp \left(-\frac{M_{\mathrm{BH}}}{x_{\mathrm{bh}, s=1 / 2} M_{c}^{T}}\right)+\exp \left(-\frac{M_{\mathrm{BH}}}{x_{\mathrm{bh}, s=1 / 2} M_{\tau}^{T}}\right)+3 \exp \left(-\frac{M_{\mathrm{BH}}}{x_{\mathrm{bh}, s=1 / 2} M_{b}^{T}}\right) \\
\left.+3 \exp \left(-\frac{M_{\mathrm{BH}}}{x_{\mathrm{bh}, s=1 / 2} M_{t}^{T}}\right)\right]+0.963 \exp \left(-\frac{M_{\mathrm{BH}}}{x_{\mathrm{bh}, s=1} M_{g}^{T}}\right), \quad(3.5)
\end{array}
$$

with

$$
M_{i}^{T}=\frac{1}{8 \pi G m_{i}}
$$

where $M_{i}^{T}$ 's are the mass of the black holes whose temperature is equal to the mass $m_{i}$ of the respective standard model particle labeled by $i, i \in(\mu, u, d, s, c, \tau, b, t, g)$ for muon, up quark, down quark, strange quark, charm quark, tau, bottom quark, top quark and gluon respectively, $x_{\mathrm{bh}}=\frac{E}{T_{\mathrm{BH}}}$, and $x_{\mathrm{bh}, s}$ is the location of the peak of the emitted instantaneous power spectrum i.e the dimensionless energy $x_{\mathrm{bh}}$ of the peak of the distribution, $\psi_{s}\left(x_{\mathrm{bh}}\right)=\frac{\gamma_{s}\left(x_{\mathrm{bh}}\right) x_{\mathrm{BH}}^{3}}{e^{x_{\mathrm{bh}}-(-1)^{2 s}}}$ with $\gamma_{s}\left(x_{\mathrm{bh}}\right)=\Gamma_{s}(E) / 27 G^{2} M^{2}$. The value of $x_{\mathrm{bh}, s}$ for $\mathrm{s}=0,1 / 2$, and 1 is $2.66,4.53$, and 6.04 respectively [42]. Thus, a standard model particle is emitted from a black hole in appreciable amount when the temperature of the black hole is such that the peak of the instantaneous power emission from the black hole is of the order of the mass of that standard model particle i.e. $x_{\mathrm{bh}, s} \sim \frac{m_{i}}{T_{\mathrm{BH}}} \Rightarrow x_{\mathrm{bh}, s} M_{i}^{T} \sim M_{\mathrm{BH}}$. For massless particles or for particles with mass much smaller compared to the black hole temperature, the contribution to particle emission is given by the integral of $\psi_{s}\left(x_{\mathrm{bh}}\right)$ over all energy, which is $6.89,3.68$ and 1.56 for $\mathrm{s}=0,1 / 2$ and 1 particles respectively [59]. The normalization of $f\left(M_{\mathrm{BH}}\right)$ is chosen such that the contribution of massless particles i.e photons and neutrinos add upto 1. In eq. 3.5, we have assumed that photons, electrons, positrons and neutrinos are freely emitted which holds true for the 
calculations in this paper, since we consider black holes with $T_{\mathrm{BH}}>m_{e}$. The contribution from photons $(2 \times 0.06)$, neutrinos $(6 \times 0.147)$ and $e^{-}, e^{+}(4 \times 0.142)$, adds upto 1.569.

We can see from Eq. 3.5 that quarks and gluons dominate the emission spectra when temperature of black hole is within an order of magnitude of their mass. Almost half of their energy is converted to photons, electrons and positrons while the other half is mostly converted to neutrinos (see Fig. 4 of [60]). These secondary electromagnetic particles have to be taken into account when calculating the spectral distortion signals.

\subsection{Calculations and results}

We us the absorption coefficient $\Gamma_{s}\left(E, s, M_{\mathrm{BH}}\right)$ provided in [59] to obtain the spectrum of primary particles emitted from the black hole. We have taken into account the change in the mass of the black hole (and therefore its evolving temperature and emitted instantaneous power) as it evaporates. To take into account the secondary emission from primary particles directly emitted from the black hole, we use the tabulated result of [60] with electroweak corrections. The authors of [60] have provided fluxes of secondary products after hadronization $\left(\gamma, e^{-} e^{+}\right.$, neutrinos, hadrons) as a function of energy for injection of different standard model particles for initial energies of the particles between $5 \mathrm{GeV}$ and $100 \mathrm{TeV}$ using PYTHIA [61]. We include the decay of unstable hadrons like pions and neutron to stable hadrons, which happens on time scales much smaller compared to the Hubble time in the redshift range of interest to us, in our calculation. For black hole evaporation, the branching fraction to different standard model particles is given by Eq. 3.5. We superimpose the secondary flux from all standard model channels to obtain the total secondary flux from black hole evaporation. We ignore the energy going into neutrinos and stable hadrons. This is a good approximation since the neutrinos only interact weakly with the baryons and photons and deposit negligible energy. The error we make by ignoring the energy going into protons and antiprotons is $\lesssim 15 \%$. We leave the inclusion of proton and antiproton interactions with the baryon-photon plasma to future work. For black holes with mass $M_{\mathrm{BH}} \approx 10^{13} \mathrm{~g}$, which decay in the y-era (at redshift $\sim 7000$ ), the temperature of the black hole is $\approx 1 \mathrm{GeV}$ and the average energy of quarks and gluons that are emitted is of the order of few GeV. For black holes decaying at earlier redshifts (i.e. lower black hole mass), the temperature of the black hole and the energy of the emitted particles is even higher. The tabulated data in [60] is therefore sufficient for the black holes considered in this paper.

In Fig. 1, the fraction of the mass of the black hole yet to be evaporated and the corresponding temperature of the black hole is plotted as a function of redshift for different initial mass. A black hole of mass $10^{13} \mathrm{~g}$ decays around $z \approx 7000$ while $10^{12} \mathrm{~g}$ mass black hole decays around $z \approx 2 \times 10^{5}$. In Fig. 2 , we plot the instantaneous spectra, after hadronization of quarks/gluons and decay of unstable particles, of emitted electromagnetic particles for black holes with mass $10^{12} \mathrm{~g}$ and $10^{13} \mathrm{~g}$, at redshift $z=2 \times 10^{5}$. At this redshift the $10^{13} \mathrm{~g}$ mass black holes are completely intact as $t<t_{\mathrm{BH}}\left(\right.$ or $\left.z>z_{B H}\right)$. Most of the photons, electrons and positrons are found around $\sim 100 \mathrm{MeV}$. This is due to the pion decay to photons and electrons- 


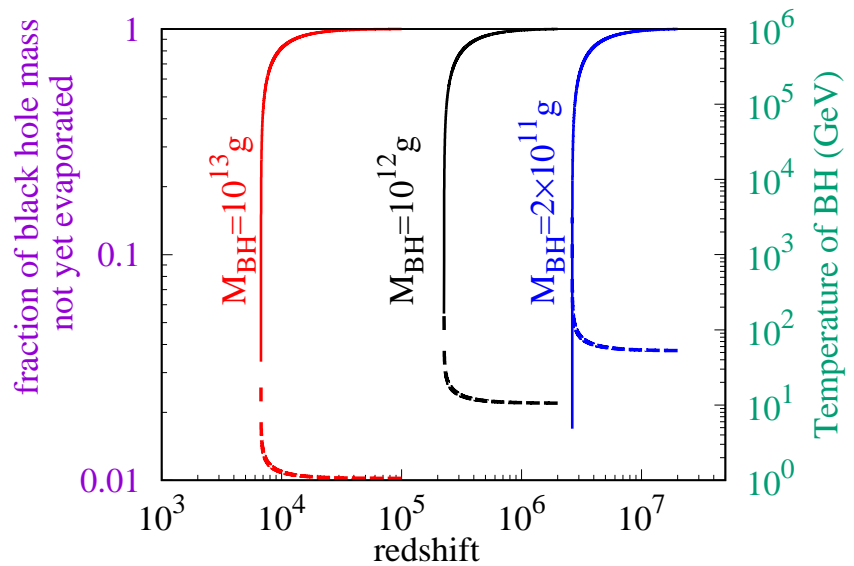

Figure 1: Fraction of mass of black holes yet to be evaporated (solid lines) and their temperature (dashed line) as a function of redshift.

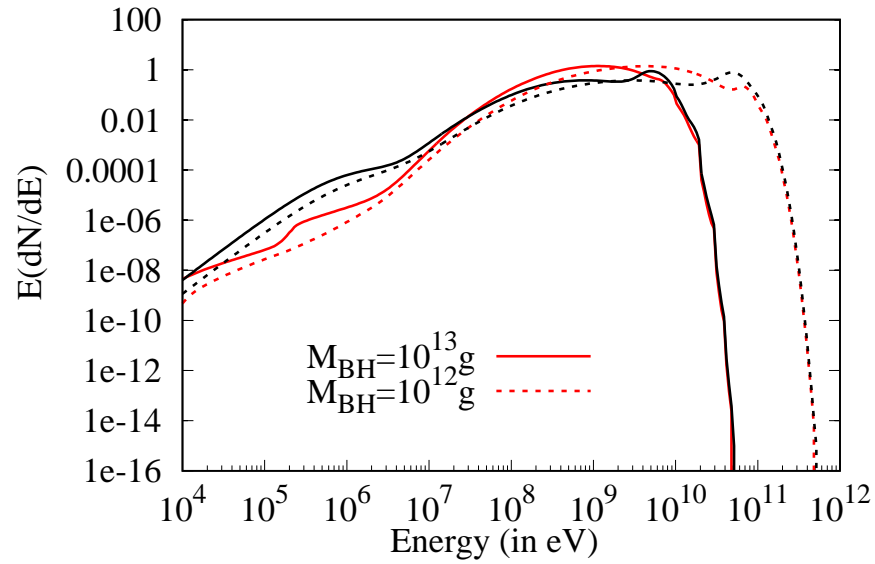

Figure 2: Instantaneous spectrum of photons (red) and electron-positron(black) pairs with number of particles per energy $(\mathrm{dN} / \mathrm{dE})$ from evaporating primordial black holes as a function of energy at redshift $z=2 \times 10^{5}$ for two different mass of black holes. At $z=2 \times 10^{5}, 10^{13} \mathrm{~g}$ mass black holes are mostly intact. All contributions, primary as well as secondary particles after hadronization and decay of unstable particles, are included.

positrons after hadronization. Charged pions first decay to muons which subsequently decay to electrons and positrons, while neutral pions decay to photon pairs. Therefore, the average energy of the electrons and positrons is slightly less compared to the photons. The bump at high energies, corresponding to $\sim x_{\mathrm{bh}, \mathrm{s}} T_{\mathrm{BH}}$, just below the exponential fall-off is due to the primary emission of photons, electrons, and positrons. In the high energy tail, electron and photon spectrum converge as the emission becomes close to thermal.

In Fig. 3, we show the spectral distortions from evaporating black holes calculated 


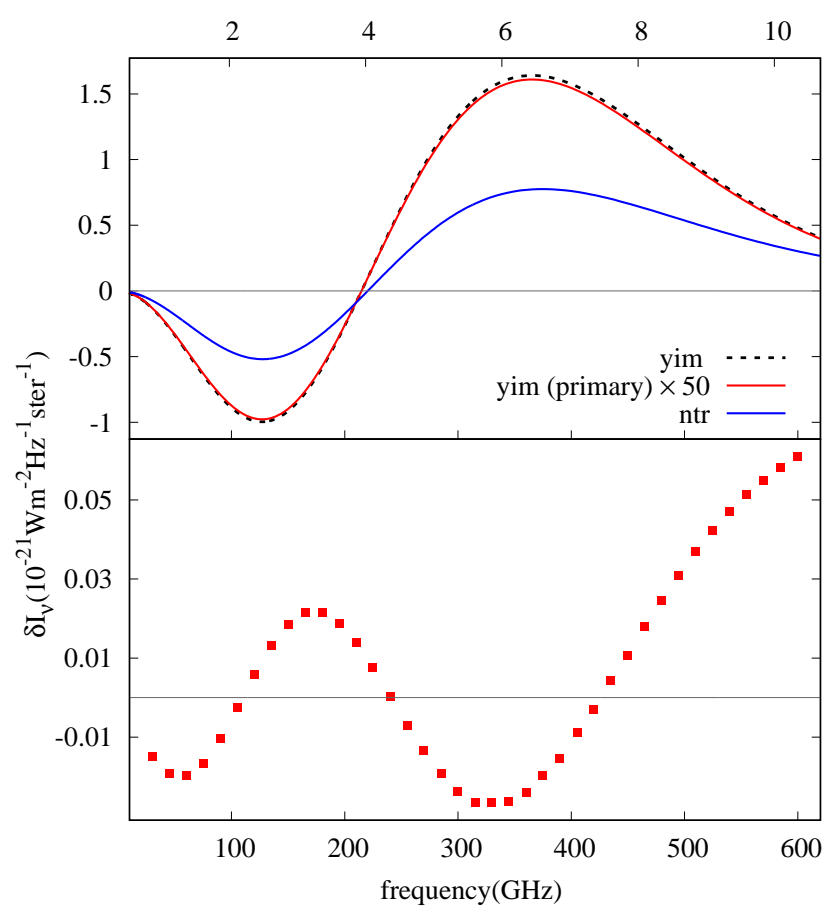

(a)

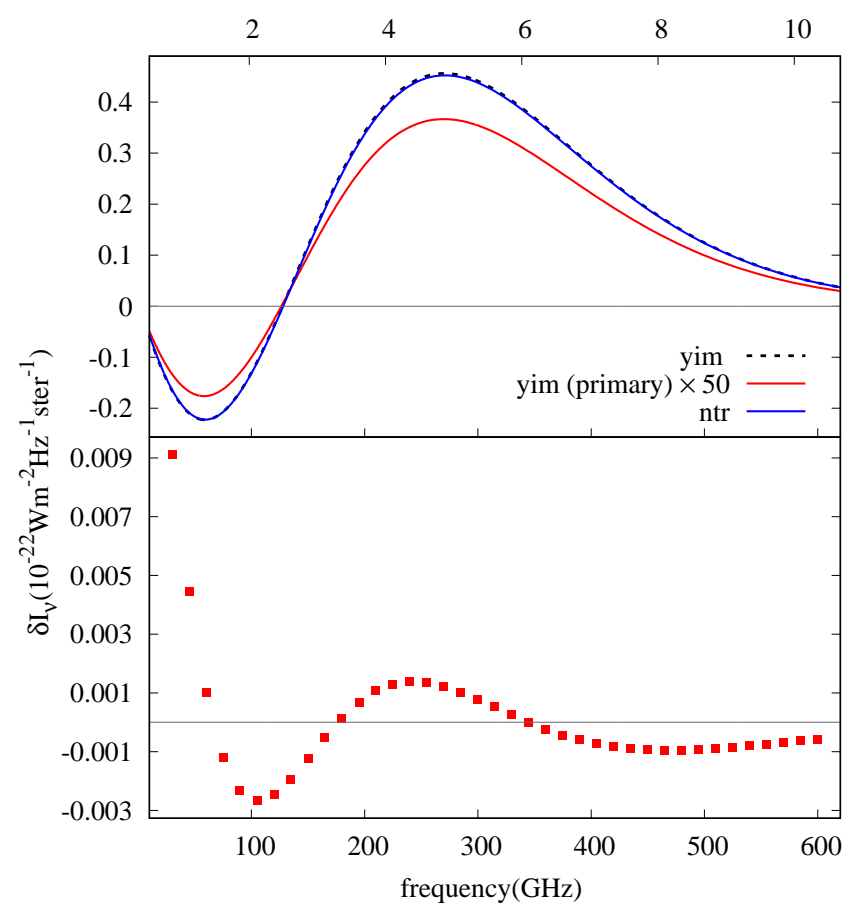

(b)

Figure 3: Spectral distortions from evaporating primordial black holes with mass $M_{\mathrm{BH}}=$ $10^{13} \mathrm{~g}$ (left) and $M_{\mathrm{BH}}=10^{12} \mathrm{~g}$ (right) for $f_{P B H}=10^{-3}$. The bottom panel shows the difference between the $n t r$ spectrum and the yim fit to the $n t r$ spectrum.

by evolving the high energy particle cascade until all energy is dissipated and the spectral distortion shape is frozen. For reference we also show the spectral distortions obtained in the yim approximation. We also plot the spectral distortion signal with just the primary photon contribution in the yim approximation (used in most of the previous works). There is a factor $\approx 50$ amplification coming by just taking into account the fact that particles other than the photons are also emitted. From Eq. 3.5, we can check that taking into account emission of all standard model particles (with the exception of top quark, W, Z bosons and higgs) gives $f\left(M_{\mathrm{BH}}\right) \sim 12$ while the contribution from primary photons is just 0.12 . Nearly half of the energy of emitted quarks and gluons is converted to secondary electromagnetic particles, with rest of the energy lost to neutrinos [60], which explains the factor 50 amplification. The actual non-thermal distortion shape for $10^{13} \mathrm{~g}$ black hole has a longer tail due to the high energy photons (being created at $z \lesssim 10000$ ) compared to the yim-approximation which makes the amplitude of the signal at the maximum (as well as minimum) of the distortion comparatively smaller. This can also be seen from the residual signal after subtracting best fit $y i m$ (i.e. trying to approximate the actual $n t r$ distortion with a $y+i+\mu$ distortion fit) signal from the $n t r$ signal. The details of the fitting procedure are described in [31]. For $10^{12} \mathrm{~g}$ and less massive black holes, the CMB spectral distortions (being created at $z \sim 10^{5}$ ) thermalize and converge to yim distortions. 


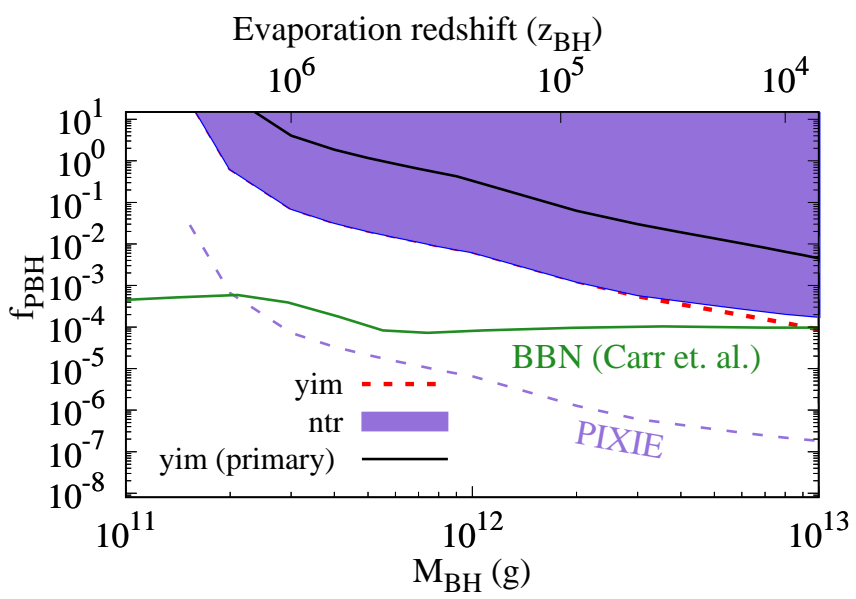

Figure 4: Constraints on the initial energy density in black holes (as a fraction $f_{\mathrm{PBH}}$ of total stable cold dark matter) as a function of mass. The projection for PIXIE-like [9] experiment with constraints stronger by a factor of 1000 is also shown. The BBN constraints are taken from Fig. 9 of [44], which is adapted from [56]. The evaporation redshift $\left(z_{\mathrm{BH}}\right)$ of the black hole is defined as the redshift at which the mass of the black hole has reduced by a factor of e $(=2.718)$. We assume monochromatic black hole mass function. The shaded region is excluded by COBE-FIRAS data.

In Fig. 4, we plot the spectral distortion constraints on the fraction of energy density in the primordial black hole as a function of the mass of the black hole. We define the fraction of primordial black holes w.r.t CDM,

$$
f_{P B H}=\frac{\rho_{P B H}\left(M_{\mathrm{BH}}\right)}{\rho_{\mathrm{cdm}}},
$$

where $\rho_{\mathrm{cdm}}$ is the stable cold dark matter energy density and $\rho_{P B H}\left(M_{\mathrm{BH}}\right)$ is the primordial black hole energy density with initial mass $M_{\mathrm{BH}}$. To derive the constraints, we have used the COBE-FIRAS [20] data. We fit the COBE-FIRAS residuals to the CMB spectral distortion templates calculated for primordial black holes of different mass [32]. Following COBE-FIRAS procedure to derive constraints on $y$ and $\mu$ distortions, we also take into account the possible deviation of the actual CMB blackbody temperature from $2.725 \mathrm{~K}$ and the Galactic foregrounds. For higher mass black holes (decaying at smaller redshifts), the ratio of energy density injected into the CMB to that of the energy density of the CMB increases as the CMB energy density $\propto(1+z)^{4}$ while $\rho_{\mathrm{BH}} \propto(1+z)^{3}$. Therefore, the spectral distortion constraints get stronger for higher mass black holes. At redshifts of $z \approx 2 \times 10^{6}$, photon number changing processes become effective [25] and constraints become exponentially weaker. Constraints from the non-thermal distortion diverge from the thermal distortions at higher mass as thermalization is less efficient at lower redshifts where the higher mass black holes evaporate. We also show the projections for PIXIE [9] and current BBN constraints from $[56,62]$. 


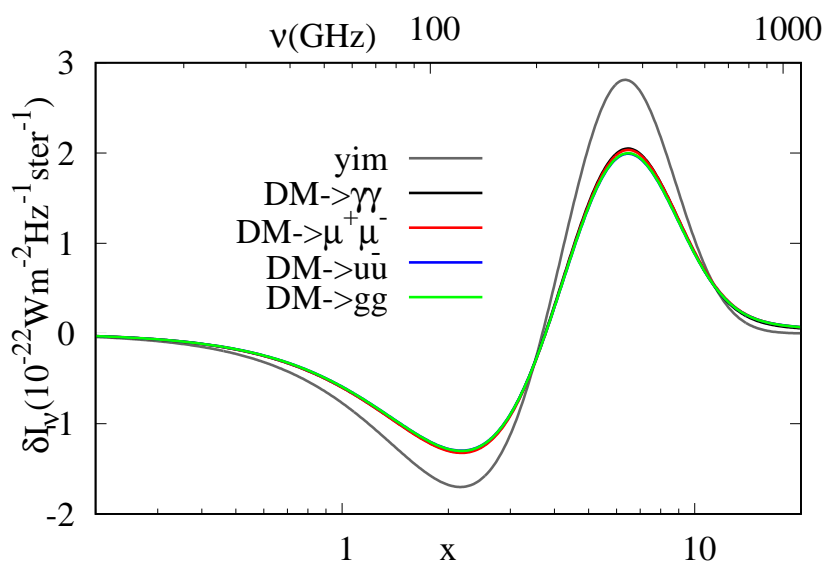

Figure 5: Spectral distortions for $10 \mathrm{GeV}$ dark matter decaying to photon, muon, up quark and gluon pairs. For photon channels $f_{X}=0.0003$. For other channels, $f_{X}$ is scaled such that total electromagnetic energy injection in these cases is equal to that of decay to photons. The decay redshift is $z_{X}=20000$. For comparison, we show the spectral distortion in the yim approximation assuming all energy goes into heating.

\section{Dark matter decay to standard model particles}

In this section, we expand the results of [32] to additional decay channels. We consider dark matter decay to neutral pions, neutral pion-photon pair, muon-antimuon pair, and charged pions for dark matter mass $300 \mathrm{MeV} \lesssim m_{X} \lesssim 2 \mathrm{GeV}$. At around $m_{X} \sim 600 \mathrm{MeV}-$ $1 \mathrm{GeV}$, free quarks and gluons are emitted instead of composite pions. The quarks and gluons after hadronization produce a spectrum of electrons, positrons, photons, neutrinos and stable hadrons. We ignore the energy going into stable hadrons (protons/antiprotons, deuterium/anti-deutrium, Fig 4 of [60]) since interactions of hadrons with baryon-photon plasma is not included in our code at present. The amount of energy going into stable hadrons depends on the channel and can be a maximum of $\sim 25 \%$ of the energy going into electrons and photons for the pure up, down, and charm quarks and gluon channels and much smaller in other channels. Our constraints are therefore slightly conservative for these channels. We plan to include this in the future. We use PYTHIA results (electromagnetic spectrum only) [60] for the spectrum of the standard model particles from dark matter decay. We can write the energy density $(E)$ injection rate as,

$$
\frac{d E}{d t}=\frac{f_{X} \rho_{\mathrm{DM}}}{\tau_{X}} \exp \left(-t / \tau_{X}\right)
$$

where $\tau_{X}$ is the particle lifetime, $z_{X}$ is the redshift at proper time $t=\tau_{X}, \rho_{\mathrm{DM}}(z)=(1+$ $z)^{3} \rho_{\mathrm{DM}}(z=0)$ is the non-decaying dark matter energy density at redshift $z$ and $f_{X}$ is the ratio of the initial energy density of decaying dark matter to that of the non-decaying component. 


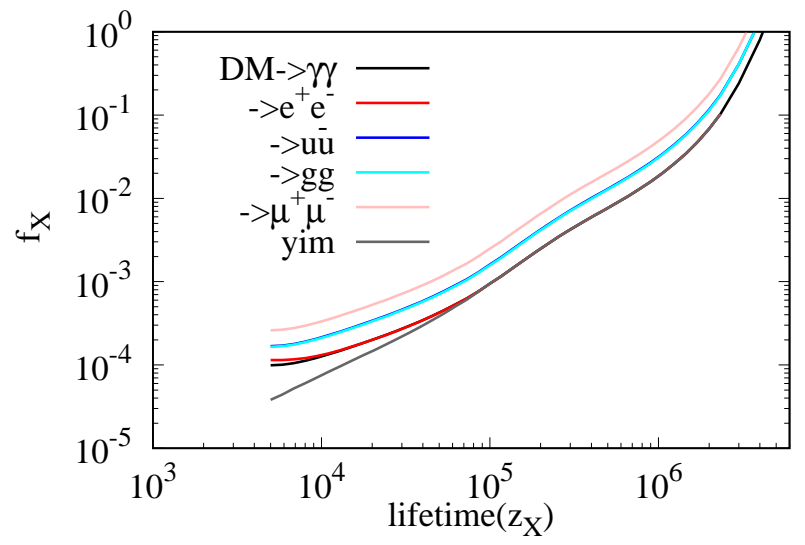

Figure 6: Constraints on fraction of decaying dark matter (with mass $\sim 1 \mathrm{GeV}$ and higher), $f_{X}$, for decay to various standard model particles as a function of lifetime. For $m_{X} \gtrsim 1 \mathrm{GeV}$ the constraints become independent of $m_{X}$.

It was shown in [32] that for dark matter of mass $\left(m_{X}\right) \gtrsim 1 \mathrm{GeV}$ decaying to electronpositron pairs or photon pairs, spectral distortions are independent of dark matter mass. This is because for photon energy $\gtrsim 1 \mathrm{GeV}$, the relevant scattering processes (photon pair production, photon-photon elastic scattering and inverse Compton scattering) are extremely fast compared to the Hubble rate. These processes immediately produce a broad, almost universal low energy photon spectrum below the threshold of these processes, irrespective of the energy of the injected photon, which makes the spectral distortions and the constraints derived from spectral distortions independent of the injected particle spectrum above a redshift dependent energy threshold. This pattern is also seen in the universal constraints on dark matter decay from big bang nucleosynthesis [18] and in the energy deposition fraction from dark matter decay or annihilation around recombination (Fig. 4 of [50]). We show in Fig. 5, the spectral distortion for a $10 \mathrm{GeV}$ dark matter decaying to different standard model particles. The shapes of spectral distortions are almost identical to that of decay to photons. The amplitude is however different for different decay channels and is proportional to the energy going into electromagnetic particles i.e photons, electrons and positrons. In particular, energy lost to neutrinos depends on the decay channel. Hence, constraints on dark matter decay to any standard model particle are sensitive to the decay channel and for dark matter mass $\gtrsim$ few $\mathrm{GeV}$ are obtained by scaling the constraints from decay to photons with branching fraction to electromagnetic particles. In Fig. 6, we show constraints on the fraction of decaying dark matter for muon, up quark and gluon channels. Others quarks have similar constraints as up quark while tau lepton channel has similar constraints as the muon channel. Leptonic channels have the weakest constraints primarily producing neutrinos while electromagnetic channels have the strongest constraints.

We also show BBN constraints for dark matter decaying to photon pairs as a rep- 
resentative case for comparison, extending the results of [4] to energies $\gtrsim \mathrm{GeV}$. The strongest constraints come from photo-dissociation of helium-4 to helium-3, resulting in helium-3 overproduction. The constraints become stronger with increasing redshifts as CMB photons have higher energy at higher redshifts. Therefore, the CMB photons can be boosted to higher energies by electrons and positrons produced in the cascade. With more number of photons above the threshold of helium- 4 destruction, $E_{\mathrm{th}}^{\mathrm{He}}$, we get stronger constraints. At even higher redshifts, electron-positron pair production threshold on the CMB photons becomes comparable to the photo-dissociation threshold of helium and the initial high energy photons get converted into electron-positron pairs instead of dissociating helium, since the number density of CMB photons is $\sim 10$ orders of magnitude larger compared to the helium- 4 nuclei. The inverse Compton scattered photons boosted by these electrons and positrons have energies less than $E_{\mathrm{th}}^{\mathrm{He}}$. Thus, even though the energy of initial injected photon maybe larger than

$E_{\mathrm{th}}^{\mathrm{He}}$, these photons are immediately degraded to lower energy photons below $E_{\mathrm{th}}^{\mathrm{He}}$ and the constraints become exponentially weaker. We also show PIXIE [9] projection which is assumed to be 1000 times stronger compared to COBE-FIRAS constraint. Thus, even though at present the BBN constraints are stronger compared to the CMB spectral distortion constraints, in the future the opposite may become true.

For $m_{X} \lesssim 2 \mathrm{GeV}$, we consider dark matter decay to neutral pions, photon-neutral pion, charged pions, and muons. These channels have been considered for the detection of dark matter through pair annihilation to gamma rays [63, 64]. We use the electron, positron, and photon spectrum provided in the references [63, 64]. In Fig. 7, we show the CMB spectral distortions for the pion and muon decay channels for $z_{X}=10000$. We can see that the shape of the distortion depends upon the decay channel since the initial injected photon, electronpositron pairs spectra are different for different decay channels. In Fig. 8 and 9, we give the constraints on dark matter decaying to neutral pions, photon-neutral pion pairs, muons, charged pions for different values of dark matter mass. For the neutral pion pair and photonneutral pion pair channels, all of the mass-energy of the dark matter is released in the form of electromagnetic energy since the neutral pion decays to two photons. For the muon and charged pion channels a significant fraction of energy is lost to neutrinos. The fraction of deposited electromagnetic energy is $\sim 35$ percent for the muon channel and $\sim 25$ percent for the charged pions, relaxing the constraints compared to the other channels.

\section{Decay of cosmic strings}

Cosmic strings are one dimensional topological defects produced in the early universe during the cosmological phase transitions that break U(1) symmetry spontaneously [65, 66]. The cosmic strings can be superconducting, carrying currents, and can radiate bursts of electromagnetic energy. The amount of energy released and the energy spectrum of particles produced in the electromagnetic bursts is a function of string tension and current $[49,67-70]$. The power emitted in electromagntic energy is [68] $P_{E M}=\Gamma_{E W} I \sqrt{\mu}$, where $I$ is the current in the string, and $\mu$ is the string tension. They can also radiate gravitational waves [71] with 

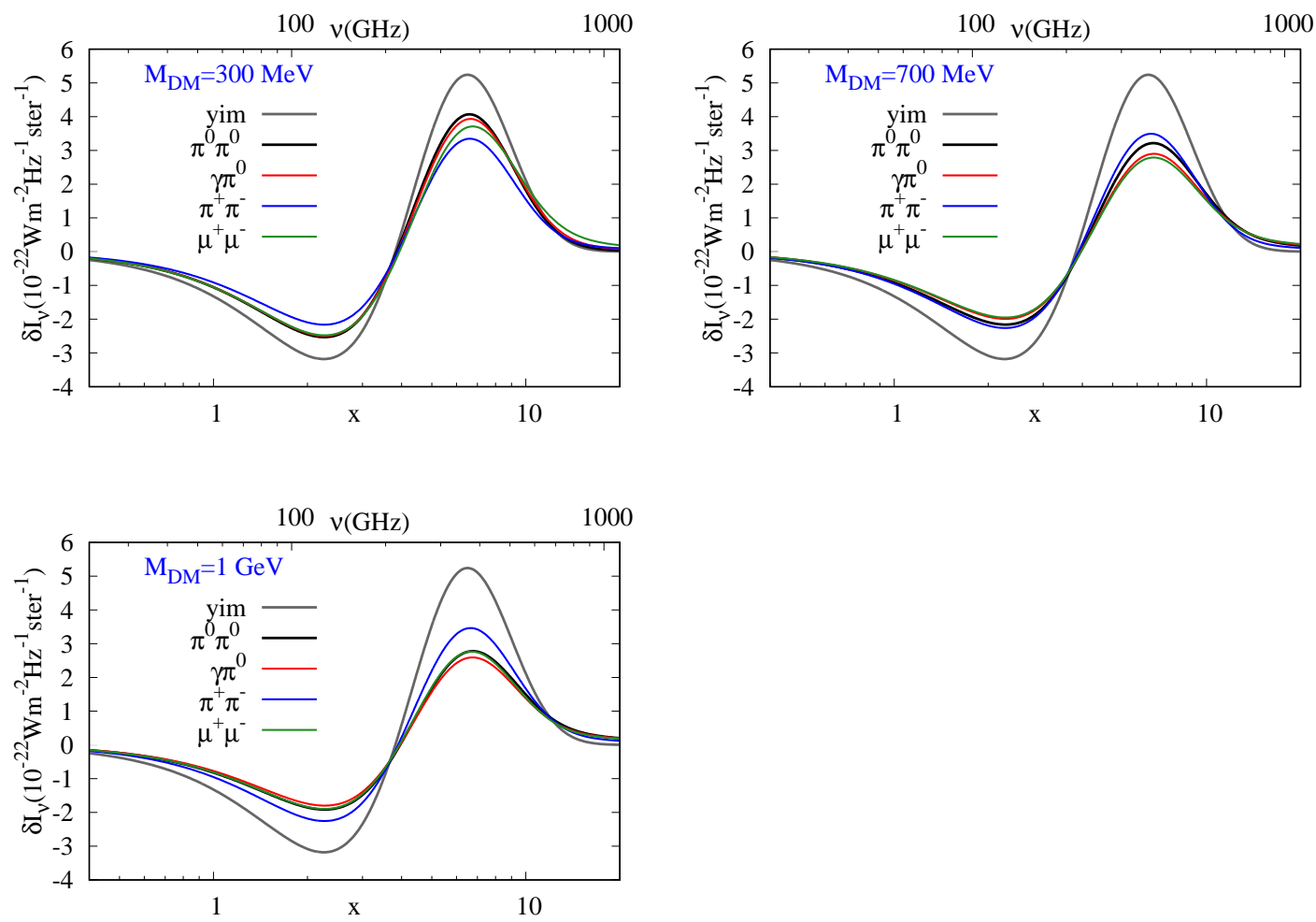

Figure 7: Spectrum for dark matter decaying to various channels with different dark matter mass, $f_{X}=0.0003$ and $z_{X}=10000$. The charged pion and muon channel curves have been scaled such that the total electromagnetic energy injection for each cases is identical. For comparison we show the spectral distortion in the yim approximation assuming all energy goes into heating.

power $P_{G W}=\Gamma_{G W} G \mu^{2}$, where $\Gamma_{G W}$ and $\Gamma_{E M}$ (in case of electromagnetic emission) depend on the shapes of string loops $[68,71]$. The radiated electromagnetic spectrum has a cutoff, which comes from demanding that the energy emitted does not backreact on cosmic string dynamics. The cutoff is given by $[68,72], \omega_{c}=2 \pi f_{c}=\mu^{3 / 2} I^{-3} l^{-1}$. The spectrum of the electromagnetic radiation is given by, $\frac{d E}{d f} \propto f^{-2 / 3}$. The crossover between electromagnetic and gravitational dominated energy loss is given by a curve in the $G \mu-I$ plane. For $I>I^{*}$, where $I^{*}=\frac{\Gamma_{G W} G \mu^{3 / 2}}{\Gamma_{E W}}$, the energy loss is mainly by electromagnetic radiation. We consider $\Gamma_{E W} \sim 10, \Gamma_{G W} \sim 50$ in this paper following [72]. These numbers are derived for string trajectories found in [73] for non self-intersecting strings.

We consider decay of cosmic strings with short-lived loops following [72] as a matter of simplicity. The rate of shrinkage is given by,

$$
\frac{d l}{d t}=-\Gamma_{e f f}
$$

where $\Gamma_{e f f}=\Gamma_{G W} G \mu+\Gamma_{E M} \frac{I}{\sqrt{\mu}}$. Assuming the length of loop at formation time $t_{i}$ to be $\alpha t_{i}$, 

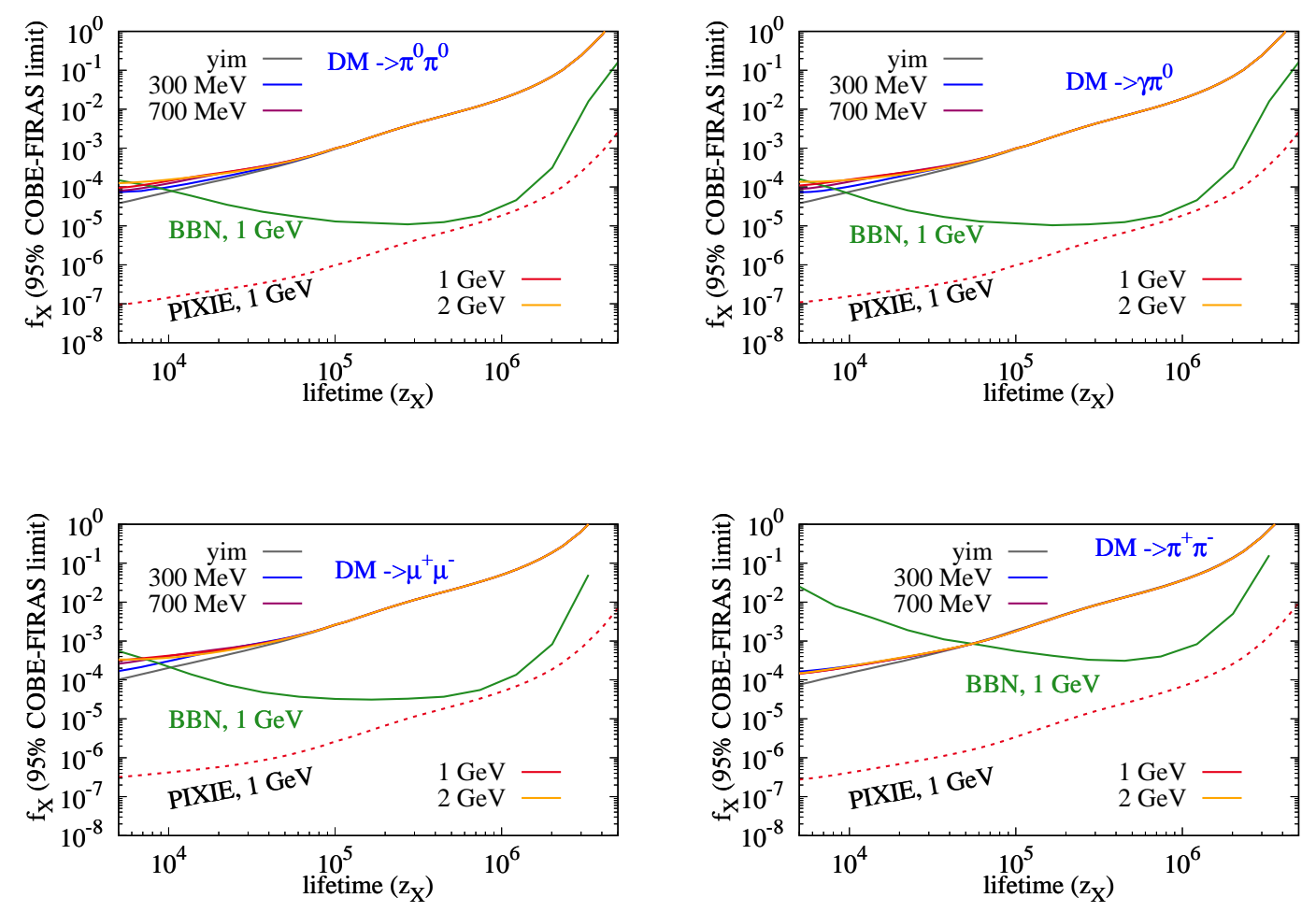

Figure 8: 95\% COBE-FIRAS upper limits on $f_{X}$ for dark matter decay to different particles for different $m_{X}$ as a function of decay redshift $z_{X}$. Also shown are the constraints assuming all energy going to yim distortions. We also show BBN constraints and PIXIE projection for a representative case of dark matter decay with mass $1 \mathrm{GeV}$.

the length of loop decreases as,

$$
l(t)=\alpha t_{i}-\Gamma_{e f f}\left(t-t_{i}\right)
$$

The lifetime of a loop is given by, $\tau=\frac{\alpha}{\Gamma_{e f f}} t_{i}$. For a short-lived loop, $\alpha=\Gamma_{e f f}$. The formation rate of loops at time $\mathrm{t}$ is given by [72], $\frac{d n}{d t} \sim \frac{1}{\gamma^{2} \alpha t^{4}}$ while number density of decaying loops for small loops is given by $n_{d}(t) \sim \frac{1}{\gamma^{2} \alpha t^{3}}$ with $\gamma=0.27$ in the radiation dominated era. The electromagnetic energy density injected per unit time is given by,

$$
\frac{d Q}{d t}=P_{E M} n_{d}(t)
$$

The spectral distortion constraints obtained in [72] assume the distortions to be of thermal nature regardless of the spectrum of injected energy. In this work, we will use the information of full injected spectrum to calculate the distortion spectrum. The photons emitted by strings can have higher or lower energy compared to the CMB photons. For a high current, a string can radiate a lot of soft photons. Therefore, we have to take into account photon 

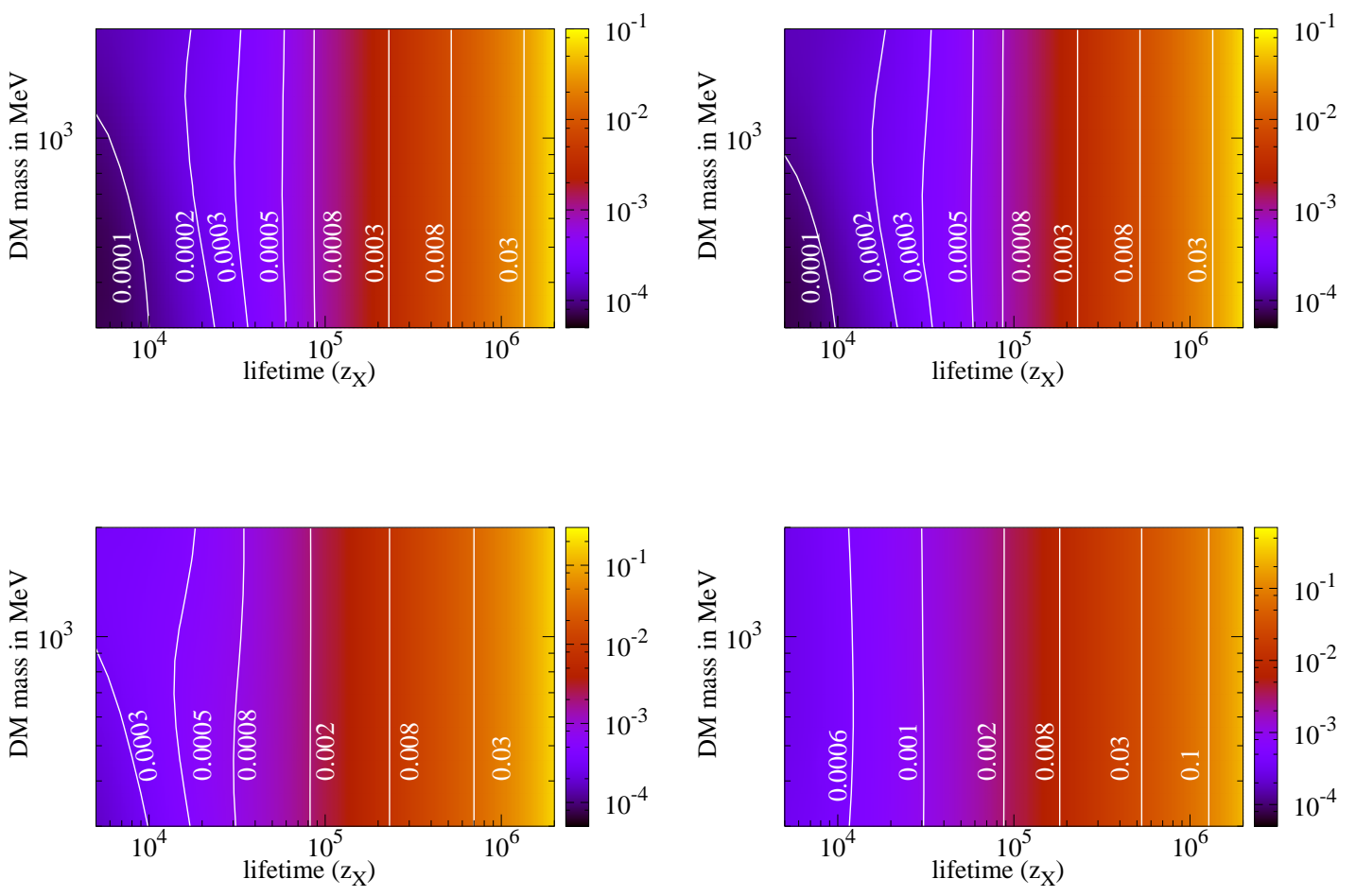

Figure 9: $95 \%$ COBE-FIRAS upper limits on $f_{X}$ for dark matter decay to neutral pions (upper left), photon-neutral pion pairs (upper right), muons (lower left), charged pion pairs (lower right) in the $z_{X}-m_{X}$ plane.

injection in the form of low energy photons (lower than the average CMB photon energy). For photon injection with $0.1 \lesssim x \lesssim 10$, where $x=\left(\frac{E_{\gamma}}{T_{\mathrm{CMB}}}\right)$, Compton scattering is the dominant process $[25,74]$. For $\mathrm{x}<0.1$, photon non-conserving processes Bremsstrahlung (BR) and double Compton scattering (DC) are important [24, 25, 74]. The soft photons are absorbed through these processes, heating the electrons. Therefore, for these low energy photon injections, we will get yim distortions, while for photon with energy $0.1 \lesssim x \lesssim 10$, distortion shape is much richer [74]. The transition from Compton scattering dominated regime to BR, DC dominated region can be seen in Fig. 3 of [74] and is in reality not sharp but gradual. In our calculations, we assume a sharp crossover between pure photon non-conserving processes (BR and DC) and Compton scattering at $\mathrm{x}=0.01$ to simplify calculations. This is a good approximation as at $x \lesssim 0.01$, the $\mathrm{BR}$ absorption rate is faster compared to the Hubble rate (Fig. 3 of [25]), implying almost complete absorption of these photons. In our approximate treatment, photons at $\mathrm{x}>0.01$ are evolved with Compton scattering which after crossing $\mathrm{x}=0.01$ boundary are converted to heat. This is a good approximation for COBE-FIRAS data, where we observe CMB spectrum only at $x>1.2$. We have checked that the spectral distortion shapes for $x>1$ are not sensitive to the choice of this boundary. 


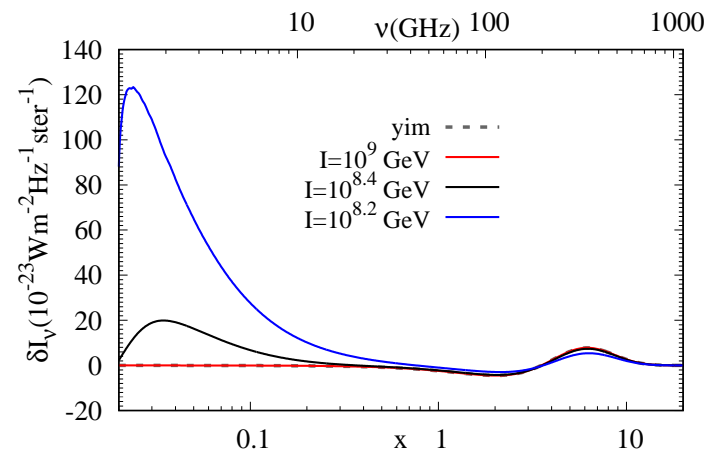

(a)

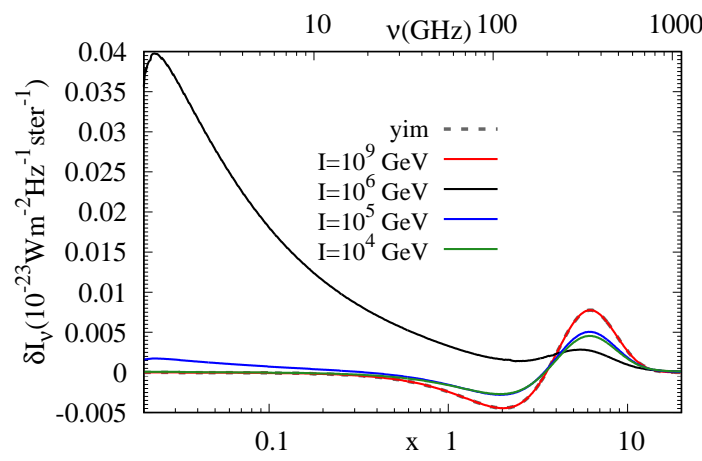

(b)

Figure 10: Comparison of spectral distortion shape with yim approximation with constant dimensionless string tension (a) $G \mu=10^{-8.6}$, (b) $G \mu=10^{-11.6}$ and varying currents.

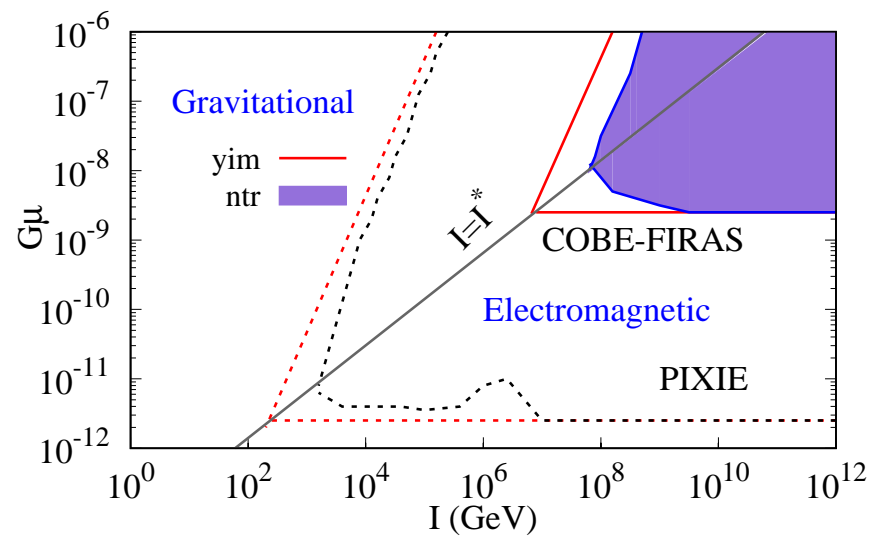

Figure 11: Exclusion plot for dimensionless string tension vs current for COBE-FIRAS [20] (black shaded region) and projections for PIXIE [9] (dashed lines). For the string parameters corresponding to the bump in PIXIE projections at $I \approx 10^{6} \mathrm{GeV}$, photons are emitted in the energy range with $0.01<x<1$. In this energy range, bremsstrahlung process is inefficient and photons have high chance to survive rather being absorbed and converted to heat, making constraints significantly weaker, since most of these photons are out of the CMB (COBEFIRAS) band.

In Fig. 10, we compare the actual spectral distortion shape and yim shape for photon injection from EM-dominated string decay with constant tension and different currents. For $I \gtrsim 10^{9} \mathrm{GeV}$, the emitted soft photons $(x<0.01)$ heat up the electrons immediately. For slightly smaller currents, photons are produced in the range $0.01<x<$ 10, which have higher chance to survive while giving a small fraction of energy to heat by 
absorption and through Compton scattering. This relaxes the COBE-FIRAS [20] constraints on allowed string tension as shown in Fig. 10a and 11 and produces the big bump at $I=$ $10^{6} \mathrm{GeV}$ in the PIXIE projections. For still lower current, photons with relativistic energies are produced (Fig. 10b) which give distortions with lower amplitude and significantly more energy in the high energy tail compared to the yim distortions. In Fig. 11, we give the spectral distortion constraints and projections for PIXIE experiment assuming a factor of 1000 improvement in sensitivity for both EM- and GW-dominated string decay over COBEFIRAS. For reference, we also show the constraints in the yim approximation i.e. assuming all radiated energy goes into heat. We see that the actual constraints are significantly relaxed and yim approximation can be off by almost an order of magnitude. For a given value of current, $I$, as we increase the string tension, the fraction of decay energy going into gravitational waves (photons) increases (decreases) and the CMB spectral distortions constraints become weaker. The high tension part of the parameter space is strongly constrained by the limits on the stochastic gravitational wave background region from the pulsar timing arrays [72].

\section{Conclusions}

In this work, we have studied the spectral distortion constraints from evaporation of primordial black holes, dark matter decay to standard model particles and decay of cosmic strings. We solve the high energy particle cascade from black hole evaporation, taking into account secondary particles produced from hadronization of emitted primary particles, and obtained constraints which are stronger by almost a factor of 50 compared to the previous works which ignored the secondary particles. Primordial black holes of mass $\sim 10^{13} \mathrm{~g}$ (decaying at $z \lesssim 10^{4}$ ) inject relativistic particles in the background baryon-photon plasma, which results in relativistic non-thermal spectral distortions having higher intensity in the high energy tail compared to the non-relativistic thermal (yim) spectral distortions. The lower amplitude of spectral intensity relaxes the constraints compared to the yim approximation by a factor of 2 . For black hole of mass $10^{12} \mathrm{~g}$ and below, the spectral distortions thermalize to $\mu$-type distortions.

We explicitly show that for decaying dark matter with mass of the order of 10 $\mathrm{GeV}$ and above, the spectral distortion shape is independent of dark matter mass and the constraints for decay to various standard model particle channels is simply a function of branching fraction to electromagnetic particles. For sub-GeV mass dark matter, the spectral distortions are non-universal having a characteristic shape that is sensitive to the decay channel as well as the mass of the dark matter particle, and can deviate from yim approximation by a factor of 2 to 3 in amplitude in the COBE-FIRAS frequency band.

We also provide constraints on superconducting cosmic strings in the string tension and current plane, taking into account the emitted photon spectrum which had been ignored in previous studies. We show that the photon emission with relativistic energy and in the CMB band has palpable consequences for both COBE-FIRAS and PIXIE spectral distortion constraints. Taking the actual shape into account relaxes the constraints by almost an order of magnitude in the region of the parameter space where photons with energy comparable or 
larger than the average CMB photon energy are being emitted. The shape of the spectral distortion is sensitive to the tension and current of the cosmic string.

The spectral distortions in the $y$-type and $i$-type era $\left(z \lesssim 10^{5}\right)$ carry rich information about the potential new physics beyond the standard model of particle physics. This information is hidden in the characteristic shapes of the CMB spectral distortions and can, with precision measurements of the CMB spectrum, distinguish the particular new physics responsible for creating the distortions. The CMB spectral distortions, if detected (other than the Sunyaev-Zeldovich effect which has already been detected), can thus be used to measure the parameters of the new physics responsible for them, such as the branching fractions and mass

of the decaying dark matter, mass function of primordial black holes, and tension and current of the cosmic strings.

\section{Acknowledgements}

This work was supported by Max Planck Partner Group for cosmology of Max Planck Institute for Astrophysics Garching at Tata Institute of Fundamental Research funded by MaxPlanck-Gesellschaft. This work was also supported by Science and Engineering Research Board (SERB) of Department of Science and Technology, Government of India grant no. ECR/2015/000078. We acknowledge support of the Department of Atomic Energy, Government of India, under project no. 12-R\&D-TFR-5.02-0200.

\section{References}

[1] J. A. Adams, S. Sarkar, and D. W. Sciama. Cosmic microwave background anisotropy in the decaying neutrino cosmology. MNRAS, 301:210-214, November 1998.

arXiv:astro-ph/9805108, [DOI], [ADS].

[2] X. Chen and M. Kamionkowski. Particle decays during the cosmic dark ages. Phys.Rev.D, 70(4):043502, August 2004. arXiv: astro-ph/0310473, [DOI], [ADS].

[3] S. Galli, T. R. Slatyer, M. Valdes, and F. Iocco. Systematic uncertainties in constraining dark matter annihilation from the cosmic microwave background. Phys.Rev.D, 88(6):063502, September 2013. arXiv:1306.0563, [DOI], [ADS].

[4] Sandeep Kumar Acharya and Rishi Khatri. CMB anisotropy and BBN constraints on pre-recombination decay of dark matter to visible particles. JCAP, 2019(12):046, Dec 2019. arXiv: 1910.06272, [DOI], [ADS].

[5] N. Aghanim et al. Planck 2018 results. VI. Cosmological parameters. ArXiv e-prints, July 2018. arXiv: 1807.06209, [ADS].

[6] de Haan et. al. Cosmological Constraints from Galaxy Clusters in the 2500 Square-degree SPT-SZ Survey. ApJ, 832(1):95, Nov 2016. arXiv:1603.06522, [DOI], [ADS].

[7] Louis et. al. The Atacama Cosmology Telescope: two-season ACTPol spectra and parameters. JCAP, 2017(6):031, Jun 2017. arXiv:1610.02360, [DOI], [ADS]. 
[8] M. Hazumi et al. LiteBIRD: A Satellite for the Studies of B-Mode Polarization and Inflation from Cosmic Background Radiation Detection. J. Low. Temp. Phys., 194(5-6):443-452, 2019. [DOI].

[9] A. Kogut, D. J. Fixsen, D. T. Chuss, J. Dotson, E. Dwek, M. Halpern, G. F. Hinshaw, S. M. Meyer, S. H. Moseley, M. D. Seiffert, D. N. Spergel, and E. J. Wollack. The Primordial Inflation Explorer (PIXIE): a nulling polarimeter for cosmic microwave background observations. JCAP, 7:025, July 2011. arXiv:1105.2044, [DOI], [ADS].

[10] Di Valentino et al. Exploring cosmic origins with CORE: Cosmological parameters. JCAP, 2018(4):017, Apr 2018. arXiv:1612.00021, [DOI], [ADS].

[11] Hanany et. al. PICO: Probe of Inflation and Cosmic Origins. arXiv e-prints, page arXiv:1902.10541, Feb 2019. arXiv:1902.10541, [ADS].

[12] Carlstrom et. al. CMB-S4. volume 51, page 209, Sep 2019. arXiv:1908.01062, [ADS].

[13] Vivian Poulin and Pasquale Dario Serpico. Nonuniversal BBN bounds on electromagnetically decaying particles. Phys.Rev.D, 91(10):103007, May 2015. arXiv:1503.04852, [DOI], [ADS].

[14] Marco Hufnagel, Kai Schmidt-Hoberg, and Sebastian Wild. BBN constraints on MeV-scale dark sectors. Part II: Electromagnetic decays. Journal of Cosmology and Astro-Particle Physics, 2018(11):032, Nov 2018. arXiv:1808.09324, [DOI], [ADS].

[15] Lindsay Forestell, David E. Morrissey, and Graham White. Limits from BBN on light electromagnetic decays. Journal of High Energy Physics, 2019(1):74, Jan 2019. arXiv:1809.01179, [DOI], [ADS].

[16] J. Ellis, D. V. Nanopoulos, and S. Sarkar. The cosmology of decaying gravitinos. Nuclear Physics B, 259:175-188, September 1985. [DOI], [ADS].

[17] J. Ellis, G. B. Gelmini, J. L. Lopez, D. V. Nanopoulos, and S. Sarkar. Astrophysical constraints on massive unstable neutral relic particles. Nuclear Physics B, 373:399-437, April 1992. [DOI], [ADS].

[18] M. Kawasaki and T. Moroi. Electromagnetic Cascade in the Early Universe and Its Application to the Big Bang Nucleosynthesis. ApJ, 452:506, Oct 1995. arXiv: astro-ph/9412055, [DOI], [ADS].

[19] Masahiro Kawasaki, Kazunori Kohri, Takeo Moroi, and Yoshitaro Takaesu. Revisiting big-bang nucleosynthesis constraints on long-lived decaying particles. Phys.Rev.D, 97(2):023502, Jan 2018. arXiv:1709.01211, [DOI], [ADS].

[20] D. J. Fixsen, E. S. Cheng, J. M. Gales, J. C. Mather, R. A. Shafer, and E. L. Wright. The Cosmic Microwave Background Spectrum from the Full COBE FIRAS Data Set. ApJ, 473:576, December 1996. arXiv: astro-ph/9605054, [DOI], [ADS].

[21] D. J. Fixsen and J. C. Mather. The Spectral Results of the Far-Infrared Absolute Spectrophotometer Instrument on COBE. ApJ, 581:817-822, December 2002. [DOI], [ADS].

[22] R. A. Sunyaev and Y. B. Zeldovich. The interaction of matter and radiation in the hot model of the Universe, II. ApSS, 7:20-30, April 1970. [DOI], [ADS].

[23] L. Danese and G. de Zotti. Double Compton process and the spectrum of the microwave background. A\&A A, 107:39-42, 1982. [ADS]. 
[24] J. Chluba and R. A. Sunyaev. The evolution of CMB spectral distortions in the early Universe. MNRAS, 419:1294-1314, January 2012. arXiv:1109.6552, [DOI], [ADS].

[25] R. Khatri and R. A. Sunyaev. Creation of the CMB spectrum: precise analytic solutions for the blackbody photosphere. JCAP, 6:38, 2012. [DOI], [ADS].

[26] Y. B. Zeldovich and R. A. Sunyaev. The Interaction of Matter and Radiation in a Hot-Model Universe. ApSS, 4:301-316, July 1969. [DOI], [ADS].

[27] A. F. Illarionov and R. A. Siuniaev. Comptonization, the background-radiation spectrum, and the thermal history of the universe. Soviet Astronomy, 18:691-699, June 1975. [ADS].

[28] C. Burigana, L. Danese, and G. de Zotti. Formation and evolution of early distortions of the microwave background spectrum - A numerical study. AEA, 246:49-58, June 1991. [ADS].

[29] R. Khatri and R. A. Sunyaev. Beyond y and $\mu$ : the shape of the CMB spectral distortions in the intermediate epoch, $1.5 \times 10^{4} \lesssim \mathrm{z} \lesssim 2 \times 10^{5}$. JCAP, 9:016, September 2012. arXiv:1207.6654, [DOI], [ADS].

[30] J. Chluba. Green's function of the cosmological thermalization problem. MNRAS, 434:352-357, September 2013. arXiv:1304.6120, [DOI], [ADS].

[31] Sandeep Kumar Acharya and Rishi Khatri. Rich structure of nonthermal relativistic CMB spectral distortions from high energy particle cascades at redshifts $z \lesssim 2 \times 10^{5}$. Phys.Rev.D, 99(4):043520, Feb 2019. arXiv:1808.02897, [DOI], [ADS].

[32] Sandeep Kumar Acharya and Rishi Khatri. New CMB spectral distortion constraints on decaying dark matter with full evolution of electromagnetic cascades before recombination. Phys.Rev.D, 99(12):123510, Jun 2019. arXiv:1903.04503, [DOI], [ADS].

[33] Gianfranco Bertone, Dan Hooper, and Joseph Silk. Particle dark matter: evidence, candidates and constraints. Physics Reports, 405(5-6):279-390, Jan 2005. arXiv:hep-ph/0404175, [DOI], [ADS].

[34] Jonathan L. Feng. Dark Matter Candidates from Particle Physics and Methods of Detection. Annual Review of Astronomy and Astrophysics, 48:495-545, Sep 2010. arXiv:1003.0904, [DOI], [ADS].

[35] Massimo Ricotti, Jeremiah P. Ostriker, and Katherine J. Mack. Effect of Primordial Black Holes on the Cosmic Microwave Background and Cosmological Parameter Estimates. ApJ, 680:829-845, Jun 2008. arXiv:0709.0524, [DOI], [ADS].

[36] Y. Ali-Haïmoud and M. Kamionkowski. Cosmic microwave background limits on accreting primordial black holes. Phys.Rev.D, 95(4):043534, February 2017. arXiv:1612.05644, [DOI], [ADS].

[37] Vivian Poulin, Pasquale D. Serpico, Francesca Calore, Sébastien Clesse, and Kazunori Kohri. CMB bounds on disk-accreting massive primordial black holes. Phys.Rev.D, 96(8):083524, Oct 2017. arXiv:1707.04206, [DOI], [ADS].

[38] S. W. Hawking. Black hole explosions? Nature, 248:30-31, March 1974. [DOI], [ADS].

[39] S. W. Hawking. Particle creation by black holes. Communications in Mathematical Physics, 43:199-220, August 1975. [DOI], [ADS]. 
[40] D. N. Page. Particle emission rates from a black hole: Massless particles from an uncharged, nonrotating hole. Phys.Rev.D, 13:198-206, January 1976. [DOI], [ADS].

[41] J. H. MacGibbon and B. R. Webber. Quark- and gluon-jet emission from primordial black holes: The instantaneous spectra. Phys.Rev.D, 41:3052-3079, May 1990. [DOI], [ADS].

[42] J. H. MacGibbon. Quark- and gluon-jet emission from primordial black holes. II. The emission over the black-hole lifetime. Phys.Rev.D, 44:376-392, July 1991. [DOI], [ADS].

[43] Hiroyuki Tashiro and Naoshi Sugiyama. Constraints on primordial black holes by distortions of the cosmic microwave background. Phys.Rev.D, 78(2):023004, Jul 2008. arXiv:0801.3172, [DOI], [ADS].

[44] Matteo Lucca, Nils Schöneberg, Deanna C. Hooper, Julien Lesgourgues, and Jens Chluba. The synergy between CMB spectral distortions and anisotropies. arXiv e-prints, page arXiv:1910.04619, Oct 2019. arXiv:1910.04619, [ADS].

[45] T. W. B. Kibble. Topology of cosmic domains and strings. Journal of Physics A Mathematical General, 9(8):1387-1398, Aug 1976. [DOI], [ADS].

[46] Edmund J. Copeland and T. W. B. Kibble. Cosmic strings and superstrings. Proceedings of the Royal Society of London Series A, 466(2115):623-657, Mar 2010. arXiv:0911.1345, [DOI], [ADS].

[47] F. R. Bouchet, P. Peter, A. Riazuelo, and M. Sakellariadou. Evidence against or for topological defects in the BOOMERanG data? Phys.Rev.D, 65(2):021301, Jan 2002. arXiv: astro-ph/0005022, [DOI], [ADS].

[48] Adam Moss and Levon Pogosian. Did BICEP2 See Vector Modes? First B-Mode Constraints on Cosmic Defects. Phys.Rev.Lett, 112(17):171302, May 2014. arXiv:1403.6105, [DOI], [ADS].

[49] E. Witten. Superconducting strings. Nuclear Physics B, 249:557-592, 1985. [DOI], [ADS].

[50] T. R. Slatyer, N. Padmanabhan, and D. P. Finkbeiner. CMB constraints on WIMP annihilation: Energy absorption during the recombination epoch. Phys.Rev.D, 80(4):043526, August 2009. arXiv:0906.1197, [DOI], [ADS].

[51] T. Kanzaki and M. Kawasaki. Electron and photon energy deposition in the Universe. Phys.Rev.D, 78(10):103004, November 2008. arXiv:0805.3969, [DOI], [ADS].

[52] T. Kanzaki, M. Kawasaki, and K. Nakayama. Effects of Dark Matter Annihilation on the Cosmic Microwave Background. Progress of Theoretical Physics, 123:853-865, May 2010. arXiv:0907.3985, [DOI], [ADS].

[53] Y. B. Zel'dovich and I. D. Novikov. The Hypothesis of Cores Retarded during Expansion and the Hot Cosmological Model. Astronomicheskii Zhurnal, 43:758, 1966. [ADS].

[54] S. Hawking. Gravitationally collapsed objects of very low mass. MNRAS, 152:75, 1971. [DOI], [ADS].

[55] B. J. Carr and S. W. Hawking. Black holes in the early Universe. MNRAS, 168:399-416, August 1974. [DOI], [ADS].

[56] B. J. Carr, Kazunori Kohri, Yuuiti Sendouda, and Jun'Ichi Yokoyama. New cosmological constraints on primordial black holes. Phys.Rev.D, 81:104019, May 2010. arXiv:0912.5297, [DOI], [ADS]. 
[57] D. N. Page. Particle emission rates from a black hole. II. Massless particles from a rotating hole. Phys.Rev.D, 14(12):3260-3273, Dec 1976. [DOI], [ADS].

[58] Tullio Regge and John A. Wheeler. Stability of a Schwarzschild Singularity. Physical Review, 108(4):1063-1069, Nov 1957. [DOI], [ADS].

[59] T. N. Ukwatta, D. R. Stump, J. T. Linnemann, J. H. MacGibbon, S. S. Marinelli, T. Yapici, and K. Tollefson. Primordial Black Holes: Observational characteristics of the final evaporation. Astroparticle Physics, 80:90-114, Jul 2016. arXiv:1510.04372, [DOI], [ADS].

[60] Marco Cirelli, Gennaro Corcella, Andi Hektor, Gert Hütsi, Mario Kadastik, Paolo Panci, Martti Raidal, Filippo Sala, and Alessandro Strumia. PPPC 4 DM ID: a poor particle physicist cookbook for dark matter indirect detection. JCAP, 2011(3):051, Mar 2011. arXiv:1012.4515, [DOI], [ADS].

[61] Torbjörn Sjöstrand, Stephen Mrenna, and Peter Skands. A brief introduction to PYTHIA 8.1. Computer Physics Communications, 178(11):852-867, Jun 2008. arXiv:0710.3820, [DOI], [ADS].

[62] K. Kohri and Jun'ichi Yokoyama. Primordial black holes and primordial nucleosynthesis: Effects of hadron injection from low mass holes. Phys.Rev.D, 61(2):023501, Jan 2000. arXiv:astro-ph/9908160, [DOI], [ADS].

[63] Alma X. González-Morales, Stefano Profumo, and Javier Reynoso-Cordova. Prospects for indirect $\mathrm{MeV}$ dark matter detection with gamma rays in light of cosmic microwave background constraints. Phys.Rev.D, 96(6):063520, Sep 2017. arXiv:1705.00777, [DOI], [ADS].

[64] Richard Bartels, Daniele Gaggero, and Christoph Weniger. Prospects for indirect dark matter searches with MeV photons. JCAP, 2017(5):001, May 2017. arXiv:1703.02546, [DOI], [ADS].

[65] A. Vilenkin. Cosmic strings and domain walls. Physics Reports, 121:263-315, 1985. [DOI], [ADS].

[66] A. Vilenkin and E. P. S. Shellard. Cosmic strings and other topological defects. Cambridge University Press, Cambridge, 1994. [ADS].

[67] J. P. Ostriker, C. Thompson, and E. Witten. Cosmological effects of superconducting strings. Physics Letters B, 180:231-239, November 1986. [DOI], [ADS].

[68] A. Vilenkin and T. Vachaspati. Electromagnetic radiation from superconducting cosmic strings. Physical Review Letters, 58:1041-1044, March 1987. [DOI], [ADS].

[69] Yi-Fu Cai, Eray Sabancilar, and Tanmay Vachaspati. Radio bursts from superconducting strings. Phys. Rev., D85:023530, 2012. arXiv:1110.1631, [DOI].

[70] Yi-Fu Cai, Eray Sabancilar, Danièle A. Steer, and Tanmay Vachaspati. Radio broadcasts from superconducting strings. Phys.Rev.D, 86(4):043521, Aug 2012. arXiv:1205.3170, [DOI], [ADS].

[71] T. Vachaspati and A. Vilenkin. Gravitational radiation from cosmic strings. Phys.Rev.D, 31:3052-3058, June 1985. [DOI], [ADS].

[72] Koichi Miyamoto and Kazunori Nakayama. Cosmological and astrophysical constraints on superconducting cosmic strings. JCAP, 2013(7):012, Jul 2013. arXiv:1212.6687, [DOI], [ADS]. 
[73] T. W. B. Kibble and Neil Turok. Self-intersection of cosmic strings. Physics Letters B, 116(2-3):141-143, Oct 1982. [DOI], [ADS].

[74] Jens Chluba. Green's function of the cosmological thermalization problem - II. Effect of photon injection and constraints. MNRAS, 454(4):4182-4196, Dec 2015. arXiv:1506.06582, [DOI], [ADS]. 